\title{
Prismatic and helical dislocation loop generation from defects
}

\author{
Lynn B. Munday ${ }^{\mathrm{a}, 1, *}$, Joshua C. Crone ${ }^{\mathrm{a}}$, Jaroslaw Knap ${ }^{\mathrm{a}}$ \\ ${ }^{a}$ RDRL-CIH-C, U.S. Army Research Laboratory, Aberdeen Proving Ground, MD, 21005
}

\begin{abstract}
Plastic deformation induced by stress concentrations near crystal defects occurs through the generation of prismatic dislocation loops (PDL). The production of PDLs leads to void growth and particle decoherence. In this work we use dislocation dynamics simulations to characterize two mechanisms for PDL formation. The first mechanism corresponds to a classical model of PDL generation from dislocation nucleation. The second mechanism considers PDL generation through cross-slip of a screw dislocation intersecting the particle. We systematically study the effect of the crystal lattice and defect type on PDL generation for both mechanisms as a function of pressure. The simulations show image stresses produced by the dislocation's interaction with the free surface of a void suppresses PDL generation. The highest PDL generation rates are found for a dislocation nucleated from a void in a body-centered cubic lattice. Our simulations also show helical coiling of screw dislocations produces a continuous emission of PDLs without the need for dislocation nucleation at pressures as low as $1.0 \mathrm{GPa}$.
\end{abstract}

Keywords: dislocation dynamics; void growth; image stress

\section{Introduction}

Plastic deformation near lattice defects is primarily dependent on locally activated modes of dislocation motion including cross-slip and nucleation [1]. These locally active dislocation mechanisms are promoted by stress concentrations arising from the lattice defects and lead to the formation of prismatic dislocation loops (PDL) around defects such as voids and misfit particles [2]. Unlike a dislocation glide loop, the Burgers vector of a PDL does not lie in the plane of the loop. This allows a PDL to displace a platelet of material relative to the defect's interface resulting in volumetric plastic deformation. In the absence of diffusion, the volumetric plastic deformation associated with the generation and emission of multiple PDL's is the primary mech10 anism for void growth or interface decoherence between a particle and the surrounding matrix material.

The specific mechanism for generating PDLs from a defect is dependent on a number of factors, such as, energy barriers to dislocation motion, nucleation and cross-slip as well as the initial dislocation density. Atomistic simulations tend to suggest PDL generation through the 15 interaction of multiple dislocations nucleated on separate glide planes [3, 4]. This type of PDL generation is favored under high strain rate loading where several dislocations are simultaneously

\footnotetext{
'Corresponding author

Email address: lynn.b.munday.civ@mail.mil (Lynn B. Munday) Preprint submitted to Elsevier
}

September 2, 2015 
nucleated on different glide planes around a defect. Still, such a mechanism may also be active at low strain rates if cross-slip of a nucleated dislocation is suppressed by energy barriers [5, 6] or free surface effects [7].

In contrast, experimentally observed PDLs created at lower strain rates around submicron sized particles [8] are assumed to form through an alternative mechanism involving dislocation cross-slip. The classical model for this type of PDL generation is due to Ashby and Johnson [2]. In this model, once a single dislocation is nucleated from the surface of a lattice defect under a hydrostatic load, a PDL will always be formed through a series of cross-slip events. Thus,

25 dislocation nucleation is considered the only barrier to PDL formation, while other barriers, such as those owing to dislocation cross-slip or free surfaces, are simply ignored. The predictions of Ashby and Johnson's model have been confirmed for PDL generation around misfit particles by means of nonlinear elastic/phase-field simulations [9] and dislocation dynamics simulations [7].

Experiments have also revealed that plastic relaxation may proceed through the formation of helical dislocations, a PDL like structure [10]. A helical dislocation appears as a long spiral of prismatic dislocation loops connected by a single screw dislocation of the same Burgers vector. Atomistic and dislocation dynamics simulations have shown the formation of helical dislocations through the interaction of a screw dislocation with prismatic dislocation loops of the same Burgers vector [11, 12]. Helical dislocations have also been proposed to form in the absence 35 of prismatic dislocation loops through vacancy assisted climb of a screw dislocation [10], a diffusion process. However, a screw dislocation under the action of the stress field produced by a misfit particle may also produce helical coils through a combination of dislocation glide and cross-slip. This alternative mechanism for PDL generation may lead to plastic relaxation near a defect without the need for dislocation nucleation.

Here, we investigate PDL generation around nanoscale defects using dislocation dynamics (DD) simulations. DD simulations are capable of accurately capturing the motion of dislocations in the vicinity of large stress gradients due to material heterogeneities [13]. Two- and threedimensional DD simulations excluding cross-slip have been used to capture the later stages of void growth through multiplication of dislocations in the crystal [14, 15]. In these simulations,

45 the number of dislocation sources in the vicinity of a void activated by its stress concentration increases with the void size. This observation may indicate higher void growth rates for large voids. The reduction in active dislocation sources for small voids is likely to cause void growth to transition from multiplication of existing dislocations to dislocation nucleation for voids below $500 \mathrm{~nm}$ [15]. For voids on the order of $50 \mathrm{~nm}$, three-dimensional DD simulations have shown that 5o locally activated modes of dislocation motion, including dislocation cross-slip and nucleation, are indispensable for PDL generation [7]. These DD simulations have also drawn attention to the importance of void image stresses for cross-slip in PDL formation at low pressures. Dislocation cross-slip at free surfaces has also been shown in three-dimensional DD simulations to be an important generator of dislocation sources available for dislocation multiplication [6, 16, 17].

The formation of PDLs through cross-slip is largely dependent on the crystal lattice which determines both the number of secondary glide planes available for cross-slip and their orientation relative to one another. Here, we consider PDL formation in face-centered cubic (fcc), body-centered cubic (bcc), and hexagonal close-packed (hcp) lattices. In atomistic simulations of hydrostatically loaded voids, the availability of cross-slip planes leads to the formation of four-

60 sided rhombus PDLs in fcc lattices [3] and hexagonal or triangular PDLs in bcc lattices [18, 4]. The structures of PDLs in hep lattices have not been identified through atomistic simulations and so we limit our study to dislocations with $\langle a\rangle$-type Burgers vectors in the close-packed directions. The four dominant slip planes containing the $\langle a\rangle$-type dislocation are the basal, prismatic 
and two different pyramidal type-I glide planes. Atomistic and DFT [19] simulations have shown

65 cross-slip of $\langle a\rangle$-type screw dislocations between the basal, prismatic and pyramidal type-I glide planes. Based on the hcp crystallography, we would expect an eight-sided PDL to form on these glide planes.

In this article, we examine PDL generation from a spherical defect, over a range of pressures, lattice types, and initial dislocation configurations. We aim to characterize each individual factor

70 affecting plastic deformation through PDL generation. In particular, we are interested in how these factors influence the cross-slip mechanism which we have found in our earlier work to be the dominant feature of PDL generation [7]. By considering the fcc, bcc, and hcp lattices, we determine the influence of availability of slip planes on cross-slip. Varying the type of spherical defect allows us to isolate the effects of dislocation interactions with free surfaces. The initial

75 dislocation structure provides estimates on two sources available for PDL generation: dislocation nucleation or preexisting dislocations. Finally, pressure provides the driving force for PDL generation around the defect and our simulations will determine its dominance on cross-slip over the other factors studied.

The article is organized as follows. In section 2 we describe the DD simulation protocol.

so Section 2.1 addresses the finite element model and section 2.2 discusses the DD simulation. We then discuss PDL generation results in section 3 Results and discussion are given separately for PDLs generated from nucleated dislocations in section 3.1. helical coiling of the infinite screw dislocation in section 3.2. and the rate of PDL formation as a function of pressure in section 3.3 We finish with a reiteration of results in the conclusions in section 4.

\section{2. Simulation Methodology}

In this work, we use dislocation dynamics (DD) simulations to capture the processes that allow PDLs to form from a dislocation interacting with a spherical defect. The material considered is aluminum with shear modulus $\mu=27 \mathrm{GPa}$, Poisson's ratio $v=0.35$, and Burgers vector $|\mathbf{b}|=2.86 \AA$. We use these material properties for all DD simulations of PDL formation in fcc, ${ }_{90} \mathrm{bcc}$, and hcp lattices. For the hcp lattice we also define $c / a=1.6236$, where $c$ and $a$ represent the vertical and lateral lattice spacing in hcp lattices, respectively. Our simulations use the DD formulation of van der Giessen and Needleman [13], where the linear elastic fields due to dislocations in an infinite bulk crystal are augmented by the linear elastic fields produced by an auxiliary boundary value problem (BVP). The boundary conditions of this BVP consist of the

95 corrective image tractions on free surfaces and far-field loads. This simulation protocol is accomplished by coupling the DD simulator ParaDiS [20] to the corrective fields obtained from a BVP solved with a parallel finite element code [21, 22].

\subsection{Finite Element Model}

The domain of our finite element model is a cube with edge length $l=4000|\mathbf{b}|$ containing a single void at the center with radius $R_{v}=100|\mathbf{b}|$. We discretize this domain with variable sized quadratic tetrahedral elements yielding a $5|\mathbf{b}|$ resolution near the void. A finely resolved mesh is needed in the vicinity of the void to resolve the image tractions and stress gradients produced by surface piercing dislocations.

Tractions are applied to the outer surface of the finite element domain commensurate with a uniform far-field hydrostatic tensile load, $\boldsymbol{\sigma}^{\infty}=p \mathbf{I}$, where $\mathbf{I}$ is the identity tensor and $p>0$. In this work pressure, $p$, is synonymous with hydrostatic tension. No image tractions are applied 
to the outer surface of the cube allowing our BVP solution to produce corrective elastic fields which approximate a single void in an infinite bulk crystal. The void acts as a stress concentrator inducing shear stresses in its vicinity. Dislocation glide is driven by the resolved shear stress on its glide plane defined as $\sigma_{b n}=\hat{\mathbf{n}} \cdot \boldsymbol{\sigma} \cdot \hat{\mathbf{b}}$, where $\boldsymbol{\sigma}, \hat{\mathbf{b}}=\mathbf{b} /|\mathbf{b}|, \hat{\mathbf{n}}=\mathbf{n} /|\mathbf{n}|$ are the infinitesimal stress tensor, the unit Burgers vector and the unit normal of the glide plane, respectively. The resolved shear stress due only to the far-field loading $\sigma^{\infty}$ is $\sigma_{b n}^{\infty}=\hat{\mathbf{n}} \cdot \boldsymbol{\sigma}^{\infty} \cdot \hat{\mathbf{b}}$ and is plotted in Figure 1 for the (a) fcc, (b) bcc, and (c) hcp slip systems that PDLs in this work will glide on. We limit our study of hcp PDLs to those formed from an $\langle a\rangle$-type Burgers vector.

The $\sigma_{b n}^{\infty}$ stress fields shown in Figure 1 do not include the effect of image tractions produced by the dislocations. The image tractions change as the dislocation structure evolves. Image effects are most pronounced for dislocations intersecting the surface where image stresses are shown to strongly influence cross-slip [16, 7]. The fine mesh needed to resolve these image stresses results in a finite element model with 500,000 degrees of freedom. In order to keep the corrective stress field in correspondence with the dislocation's configuration, the BVP must be solved with updated image traction boundary conditions at every dislocation dynamics time step. Owing to the fact that the finite element mesh and its stiffness matrix remain static over the course of our dislocation dynamics simulation, we efficiently solve our BVP by means of the MUMPS parallel direct solver [23, 24].

\subsection{Dislocation Dynamics Model}

In the ParaDiS DD simulations, dislocations are represented as discrete line segments spanning a set of nodes [20]. The maximum dislocation segment length in our simulations is $15|\mathbf{b}|$. The dislocation segments interact with one another elastically and through a line-tension approximation. The elastic interactions in ParaDiS use a nonsingular dislocation stress field [25] with 30 dislocation core radius $r_{c}=|\mathbf{b}|$. The nonsingluar elastic field is valid for a dislocation contained in an infinite bulk crystal. The total force on a dislocation is then computed by correcting the infinite body dislocation fields computed in ParaDiS through superposition with the corrective fields given by the finite domain BVP [13].

The dislocation velocities are calculated from the total dislocation force using a linear mobility function $f_{i}=B_{i} v_{i}$ where $f$ is the force, $B$ is the drag coefficient, and $v$ is the velocity. Subscript $i$ indicates the dislocation character, either edge or screw. In order to isolate the effect of the crystallography from the mobility function, our simulations use the same $B_{i}$ for all lattice types and slip planes. We assume that drag coefficients in the linear mobility function satisfy $B_{\text {screw }} / B_{\text {edge }}=2$. This ratio is based on atomistic simulations of edge and screw dislocations in

140 aluminum [26]. For our simulations, it also provides an additional benefit of slightly increasing the likelihood of producing screw oriented dislocation segments available for cross-slip. PDL generation is entirely dependent on the mobility of the screw oriented dislocation as it encircles the defect. The mobility of edge dislocations controls the rate that the fully formed PDL leaves the defect and the ratio of $B_{\text {screw }} / B_{\text {edge }}$ will have a minimal effect on PDL generation. In addi-

145 tion, the linear mobility function neglects the Peierls stress barrier for dislocation motion at low shear stresses and the bilinear relationship between stress and dislocation velocity at high shear stresses [26]. We minimize these errors by limiting our simulations to PDL formation and not the transmission of the PDL away from the defect. During PDL formation, the resolved shear stresses are maintained at a level well above the Peierls stress barrier. The bilinear mobility function is accounted for when computing PDL formation times in section 3.3 .

Dislocation cross-slip allows a dislocation to dissipate the maximum amount of plastic work from the system by allowing the dislocation to move on the glide plane that maximizes the 
area swept by its motion. Our simulations allow cross-slip of a screw dislocation to occur once the resolved shear stress on the cross-slip plane exceeds that on the current glide plane. This cross-slip criterion will maximize the energy dissipation when all glide planes have the same dislocation mobility. This is the case for the fcc and bcc lattices where all glide planes have an equivalent crystal packing and the same $B_{i}$ on each glide plane. In the hcp lattice, the $\langle a\rangle$-type dislocation cross-slips onto nonequivalent glide planes which ParaDiS treats with different mobilities [27, 28]. Our current cross-slip criterion does not account for the difference in mobilities and therefore we treat all hcp glide planes with the same mobility. By neglecting the differences in mobility, we may inadvertently allow cross-slip to occur onto planes where it should be suppressed. The cross-slip model also disregards the energy barriers required for cross-slip of partial dislocations in fcc [5, 6] and hcp [19] crystals. These errors are minimized in our simulations for the following reasons: (1) cross-slip is driven by large resolved shear stresses and (2) crossslip mainly occurs on a free surface where the activation energy has been shown to be almost negligible in atomistic and DD simulations of fcc nickel [5, 6].

We explore the availability of cross-slip planes on PDL generation by considering fcc, bcc and hcp lattices. The lattices differ in the number of secondary slip planes available for crossslip and the orientation of the cross-slip planes relative to one another. We rely on the existing 170 implementation of mobility modules for these lattices in ParaDiS [20, 27]. The family of fcc slip systems is $\{111\}\langle 110\rangle$ and only two glide-planes, $\mathbf{n}=(111)$ and $(\overline{1} \overline{1} 1)$, share $\mathbf{b}=[\overline{1} 10]$. Therefore, only one set of secondary glide planes are available for cross-slip of fcc screw dislocations. In the bcc lattice, three glide planes, $\mathbf{n}=(0 \overline{1} 1),(\overline{1} 10)$ and $(\overline{1} 01)$, share $\mathbf{b}=[111]$. In the hcp lattice, the four glide planes shown in Figure 1]d) share the $\langle a\rangle$-type Burgers vector. As

175 previously mentioned, these four hcp glide planes are not equivalent and are referred to as basal for $\mathbf{n}=(0001)$, prismatic for $\mathbf{n}=(1 \overline{1} 00)$, and pyramidal type-I for $\mathbf{n}=(1 \overline{1} 01)$ or $(\overline{1} 101)$.

A detailed description of modifications to ParaDiS in order to allow for accurate treatment of free surfaces are given in references [21, 22]. These modifications allow for dislocations intersecting a free surface to be cut with a node placed on the surface. The surface nodes are then 180 constrained to move along the surface. Surface nodes are also allowed to cross-slip in the present work if the dislocation segment is within $2^{\circ}$ of the screw orientation [7]. For the free surface created by the void, Burgers vector conservation of the cut dislocations is assured by connecting surface dislocation nodes to the center of the void via virtual dislocation segments [29, 7] These virtual dislocations do not incorporate a line tension energy and their elastic interactions with 185 conventional dislocations outside the void are canceled out by the corrective elastic fields from the BVP. We also remove the line-tension energy from the surface piercing dislocation segments to approximate the effect of the free surface. The effect of removing the line-tension energy from the surface piercing dislocation on the PDL contour is presented in section S1 of the supplementary data.

190 Dislocation nucleation is not considered in the present work and all simulations begin with an initial dislocation configuration. Two initial dislocation configurations are considered: an incipient dislocation loop and an infinite screw dislocation. In Figure 2, we show the two initial dislocation configurations placed on the bcc $(0 \overline{1} 1)[111]$ slip system. The fcc and hep simulations use the same set-up with the initial dislocation configurations placed on the (111)[110] slip system for fcc and the (0001) $1 / 3$ [11 20$]$ slip system for hcp. The red half circle represents the incipient dislocation loop with radius $R_{L}=25|\mathbf{b}|$. The infinite screw dislocation shown by the blue line is oriented at $\phi=4^{\circ}$ with respect to $\mathbf{b}$ in order to inhibit cross-slip of the bulk dislocation line. The dislocations in both initial configurations intersect the void in the vicinity of where $\sigma_{b n}^{\infty}$ 
reaches its maximum value for their slip system. This intersection point is located $R_{v} / \sqrt{2}$ from 200 the void center as indicated in Figure 2 a). At this point, the maximum resolved shear stress on the glide plane shown in Figure 1 reaches $\pm \frac{3}{4} p$.

In addition to the above void simulations, we perform simulations of an Eshelby inclusion [30]. An Eshelby inclusion also known as a misfit particle is created within a homogenous linear elastic domain when a subvolume of material undergoes uniform inelastic deformation or

205 eigenstrain. To this end, we replace the void by a sphere of aluminum with a purely dilational eigenstrain. The action of the dilational eigenstrain induces the same resolved shear stresses as the hydrostatically loaded void, see section S2 of the supplementary data for details. This includes no resolved shear stresses inside the misfit particle which is in a state of hydrostatic stress. However, unlike the void simulations, dislocations do not interact in any way with the interface 210 between the matrix and misfit particle. Therefore, dislocations are not cut at the interface but are allowed to move across it. Moreover, dislocations do not interact with the interface through image forces. In our previous work [7], the misfit particle allowed us to elucidate the effects of the free surface on PDL formation from those effects due to the far-field loading.

\section{Results and discussion}

We now present results of dislocation dynamics simulations of PDL formation around a spherical defect over a range of pressures. Both the void and the misfit particle, as well as, fcc, bcc and hcp crystal lattices are considered. Section 3.1 contains results for PDL formation from an incipient dislocation loop. This is an extension of our previous work [7] on PDL formation in fcc lattices to bcc and hcp lattices. Section 3.2 contains results for PDL formation from an infinite screw dislocation for all lattice and defect types. In section 3.3 , we discuss the rate of PDL formation for the different crystal lattices, initial dislocation configurations and defect types.

\subsection{PDL formation from an incipient dislocation loop}

In this section, we assess PDL generation from an incipient dislocation loop via the classical model of Ashby and Johnson [2]. PDLs generated in these simulations extend our previous work on fcc crystal lattices [7| to bcc and hcp crystal slip systems. In Figure 3, we plot the major stages in the process of generating a PDL in a bcc lattice for $p=2.5 \mathrm{GPa}$. Two views are given at each time-stamp labeled by the non-dimensionalized time, $\tilde{t}=t \mu / B_{\text {screw }}$, where $t$ is the simulation time, $\mu$ is the shear modulus, and $B_{\text {screw }}$ is the drag coefficient on the screw oriented portion of the dislocation [9]. The lower sequence of images is viewed normal to the dislocation's Burgers vector, $\mathbf{b}=$ [111], providing a view of the PDL contour. The upper sequence provides a profile of the PDL as it peels off from the void's surface. The dislocation making up the PDL is colored by its glide plane and the void surface is colored according to the glide plane that maximizes the resolved shear stress.

${ }_{235}$ The process of generating a PDL in the bcc lattice illustrated in Figure 3 starts from an incipient dislocation loop shown at $\tilde{t}=0$. The incipient dislocation loop intersects the void at two points creating surface piercing dislocations. The resolved shear stress created by the void causes these two surface piercing dislocation segments to glide away from one another on their current glide plane. As the loop grows and the surface piercing dislocations sweep across

240 the void's surface, they begin to experience a resolved shear stress on a secondary glide plane exceeding that on their current glide plane. The location where this occurs is indicated by the 
boundary of the gray-scaled regions. At this point, the screw oriented surface piercing dislocation switches glide planes through cross-slip and begins moving on the new glide plane. Cross-slip is illustrated by a change in the dislocation's coloring. The moment just after a cross-slip event is shown in Figure 3 at $\tilde{t}=2500$ where two short surface piercing dislocation segments have cross-slipped as indicated by their change in color.

After a series of surface cross-slip events, the final step in the formation of the PDL is shown at $\tilde{t}=4900$ where the two surface piercing dislocations meet and annhilate with one another on the $\mathbf{n}=(0 \overline{1} 1)$ glide plane, detaching the PDL from the void's surface. The completely detached PDL shown at $\tilde{t}=5100$ is driven by the resolved shear stresses away from the void in the $\mathbf{b}=[111]$ direction. This series of events is in good agreement with the model of Ashby and Johnson [2] according to which a PDL will always form from a stable incipient dislocation loop. An incipient dislocation loop is stable if the resolved shear stresses are large enough to keep it from being pulled into the void. For the void size used in our simulations, the $R_{L}=25|\mathbf{b}|$ incipient dislocation loop is stable when $p>1.4 \mathrm{GPa}$.

PDL generation from a void in a bcc lattice for a range of pressures ( $p=1.5-4.5 \mathrm{GPa})$ results in essentially the same PDL contour shown in Figure 3 at $\tilde{t}=5100$, cf. Figure S5 in the supplementary data for details. These bcc PDL contours are only slightly smaller than the ideal PDL contour indicated by the black dashed hexagon in Figure 3 The ideal contour is the PDL that would be produced if PDL generation only depended on the resolved shear stresses. This ideal contour disregards the effects of image stresses produced by the free surface or the character of the surface piercing dislocation. From these findings we conclude that PDL generation in the bcc lattice is only dependent on the resolved shear stress created by the far-field load.

In contrast to the bcc lattice, PDL generation from a void in the fcc lattice is strongly dependent on the applied pressure as shown in Figure 4(a) and (b) and our previous work [7]. For completeness, the fcc simulations from our previous work [7] are redone here using the same finite element mesh used for the bcc and hcp simulations presented in this section. This finite element discretization is slightly coarser near the void and produces an fcc PDL contour that is qualitatively the same as those produced previously [7], cf. Figure S1 in the supplementary data 270 for mesh sensitity of the fcc PDL contours.

We reiterate here the main conclusions from our previous work [7] concerning PDL formation in the fcc lattice. Two competing factors are found to control cross-slip in the fcc lattice, leading to two seperate pressure regimes of PDL formation. In the high pressure regime ( $p \geq 3.5 \mathrm{GPa}$ ), cross-slip is dominated by the resolved shear stresses created by the far-field load,

275 producing contours like that shown in Figure 4(b). This results in PDL generation that closely follows the model of Ashby and Johnson [2]. At low pressures ( $p=2.5-3.0 \mathrm{GPa})$, cross-slip is dominated by the image force produced by the dislocation's interaction with the free-surface resulting in the reduced PDL size shown in Figure 4(a). The image force indicated by the cyan arrow in Figure 4(a) is closely aligned with the secondary cross-slip plane, leading to premature 280 cross-slip at the acute corners of the PDL contour. Image stresses cause PDL generation to cease for pressures at or below $p=2.0 \mathrm{GPa}$.

Simulations of PDL generation in the hcp lattice are also shown to exhibit a pressure dependence in Figure 4(d) and (e), albeit different from that observed in the fcc lattice. Full PDLs are formed from a void in the hcp lattice starting at $p=1.5 \mathrm{GPa}$, cf. Figure S5(d) in the supple-

285 mentary data for details. In the low pressure simulation shown in Figure 4(d) for $p=2.5 \mathrm{GPa}$, a hexagonal PDL is formed where no cross-slip from the pyramidal to prismatic plane occurs. As the pressure is increased to $p=4.0 \mathrm{GPa}$, pyramidal to prismatic cross-slip occurs, producing the eight sided PDL contour shown in Figure 4(e). 
The generation of hcp PDLs is mainly driven by the applied loading in a manner similar to the bcc lattice. However, at a few select locations where cross-slip occurs, the image force will favor one secondary slip system over the other. If the resolved shear stresses due to the far-field load are sufficiently small, the imbalance created by the image force will determine the cross-slip plane. This is observed in Figure 4(d) where the direction of the image force indicated by the orange arrow favors cross-slip from one pyramidal plane to the other (green line to red or vice

295 versa). For this cross-slip event, the image force is perpendicular to the prismatic plane and does not provide any impetus for pyramidal to prismatic cross-slip. This is in contrast to the fcc lattice where the image force can only induce premature cross-slip onto a single secondary cross-slip plane.

At higher pressures, pyramidal to prismatic cross-slip is produced in the hcp lattice as demon-

${ }_{300}$ strated in Figure 4(e) for $p=4.0 \mathrm{GPa}$. This eight sided PDL contour is close to that predicted for the ideal case that only accounts for the far-field load. The relative length of each side of the hcp PDL contour is dependent on the lattice's $c / a$ ratio. For a realistic lattice with $c / a>1.5$, the pyramidal and prismatic planes become closely aligned; decreasing the amount of slip that the far-field loading would be expected to induce on the prismatic plane. It is important to note

305 that the hcp PDL contours are dependent on the dislocation mobility and cross-slip criterion. For a material like magnesium, the dislocation mobility on the type-I pyramidal plane is an order of magnitude lower than the basal and prismatic planes [31]. This lower mobility may suppress cross-slip onto the type-I pyramidal plane leading to an entirely different PDL contour.

In order to further elucidate the role of image stress on the formation of PDLs, we perform 310 a second set of simulations for PDL generation from a misfit particle for all three lattice types. The evolution of a PDL from a misfit particle in the bcc lattice is presented in Figure 5. The stress field produced by the misfit particle's dilational eigenstrain is equivalent to a void with an internal pressure of $p=2.5 \mathrm{GPa}$. In this set of simulations, the dislocation is not cut at the misfit particle interface but extends inside, creating a full dislocation loop. This results in the initial dislocation configuration shown at $\tilde{t}=0$, where the incipient dislocation loop intersects the misfit particle's interface and forms a complete loop. Inside the misfit particle, the stress field produced by the dilational eigenstrain is purely hydrostatic, yielding no force on the dislocation. Exterior to the misfit particle, the dislocation is driven by the same resolved shear stress as those produced around the void loaded in hydrostatic tension, cf. section S2 of the supplementary data 320 for details.

The dislocation is shown in Figure 5 to evolve into a PDL in much the same way as previously observed for the void simulations. At $\tilde{t}=6300$, the portion of the dislocation outside the misfit particle has grown under the resolved shear stresses produced by the misfit strain and screw oriented portions of the dislocation near the interface have cross-slipped to new glide planes. A mirror image of the external dislocation is observed to be forming on the interior of the misfit particle. At $\tilde{t}=15000$, both arms of the dislocation forming the PDLs meet and annhilate, detaching the internal and external PDLs from one another. Both PDLs are shown at $\tilde{t}=22300$, where the external PDL is being driven by the resolved shear stress away from the misfit in the $\mathbf{b}=[111]$ direction. The internal PDL remains inside the misfit particle near the interface.

Several differences in PDL generation can be observed between the void and misfit particle simulations. The most obvious is the formation of two seperate PDLs in the misfit particle simulations. The creation of two PDLs is caused by the dislocation not being terminated at the particle interface. As the applied loading causes the dislocation to grow and cross-slip outside the misfit, the portion of the dislocation inside must follow, as shown at $\tilde{t}=6300$. This results in the PDL formed inside the misfit to have an opposite line direction or Burgers vector to the 
PDL formed on the particles exterior. Since the two PDLs have opposite Burgers vectors, they are driven in opposite directions by the resolved shear stresses external to the misfit particle. The external PDL is then driven away from the misfit by these external resolved shear stresses while the resolved shear stresses drive the internal PDL into the misfit. For this reason, the internal PDL remains stuck near the misfit particles interface.

Another difference in PDL generation between the two defect types is the back and forth cross-slipping observed in the misfit particle simulations. This is indicated by the rapid color changes and rounding of the PDL contour corners in Figure 5(b). The back and forth crossslipping occurs in the region where the resolved shear stress on the primary and secondary glide plane are nearly identical. As the dislocation switches to a new glide plane that maximizes the resolved shear stress of the applied load, the dislocation-dislocation interactions may change, pulling the dislocation back to its previous glide plane. This back and forth flickering stops once the resolved shear stress on the glide planes becomes substantially different. Flickering would likely be suppressed by an energy barrier for cross-slip [5, 6].

In our work, the most important distinction between the two defect types is the absense of a pressure dependence on PDL formation in the misfit particle simulations. This allows us to correlate all pressure dependence observed in the void simulations to the image stress created by the void. To show this, we perform misfit particle simulations for the bcc, hcp and fcc lattices over a range of pressures, $p=2.5-4.5 \mathrm{GPa}$, cf. Figure S5 in the supplementary data for details. PDL generation from the misfit particle is independent of pressure and the PDL contours shown in Figures 3 (b), 4(c) and 4(f) are representative of all pressures simulated. For all misfit particle simulations, a PDL is formed once $p \geq 2.5 \mathrm{GPa}$. Below this pressure, the dislocation-dislocation interactions cause the inicipient dislocation loop to collapse onto itself and annhilate. For all lattice types, the misfit particle PDL contours come very close to matching their ideal contours. In our previous work [7], the lack of a pressure dependence in the misfit particle simulations confirmed that image stresses were responsible for the pressure regimes observed for fcc PDL generation from a void. The hcp misfit particle simulations are shown to produce slip on the prismatic plane which confirms that image stresses are responsible for suppressing cross-slip onto the prismatic plane at lower pressures in the void simulations.

\subsection{Helical coiling PDLs from an infinite screw dislocation}

In this section, we consider an alternative mechanism for PDL generation through helical coiling of a screw dislocation. The infinite screw dislocation shown in Figure 2 (b) is used as the initial dislocation configuration for this set of simulations. The infinite screw dislocation provides an ideal dislocation for evaluating the effects of image stress in two ways. First, it is stable at zero pressure and therefore can be modeled at considerably lower pressures than the incipient dislocation loop requiring $p>1.4 \mathrm{GPa}$ in order to remain stable. Lowering the pressure allows the image effects to become more pronounced. Second, since the screw dislocation pierces the void at the onset, cross-slip may occur as soon as it is favored by the resolved shear stress on the secondary glide plane. This is in contrast to the incipient dislocation loop which cannot cross-slip until it bows out far enough for the surface piercing dislocations to take on a screw orientation.

In Figure 6, we plot the evolution of the infinite screw dislocation as it forms a helical PDL around a void in the bcc lattice at $p=2.5 \mathrm{GPa}$. Two views are given at each time step labeled by the non-dimensionalized time, $\tilde{t}$. The top view provides a profile of the screw dislocation as it begins coiling from the void. The bottom view shows the developing contour of the helical PDL on the [111] void face. At $\tilde{t}=0$, the infinite screw dislocation pierces the void once as it enters

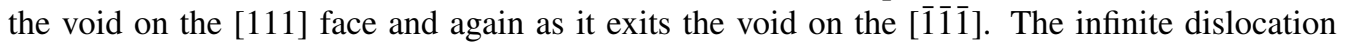


is cut at these two points and the portion inside the void is removed. At $\tilde{t}=3100$, the screw dislocation begins to coil as the resolved shear stresses drive the dislocation around the void

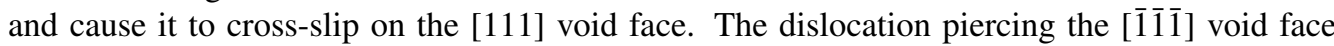
is shown to be forming coils in the opposite direction. At $\tilde{t}=4700$, a helical coil equivalent to half a PDL has been formed on each void face. Coiling of the screw dislocation is shown to continue at $\tilde{t}=7000$ as the surface piercing dislocations cross-slip around both faces of the void. At $\tilde{t}=10300$, the surface piercing dislocation has reached its initial starting point on the void surface and a full coil of the helical dislocation is produced from each face. After a full coil is created, the screw dislocation will continue to circle the void, creating additional helical PDLs. This allows a single screw dislocation piercing a void to produce several helical PDLs leading to continous void growth without nucleating new incipient dislocation loops.

The resolved shear stress acting along the length of the infinite screw dislocation shown at $\tilde{t}=0$ dies off as $r^{-3}$, where $r$ is radial distance from the void surface, cf. section S2 of the supplementary data for details. This causes the surface piercing dislocation to glide much faster than the rest of the dislocation line. The resolved shear stress drives the edge component of the dislocation coil away from the void in the direction of $\mathbf{b}$ while the screw component is driven around the void surface tracing out the PDL contour. The resolved shear stress on the [111] void face shown in Figure 1 (b) reverses sign on the [1 $1 \overline{1} \overline{1}]$ void face. This reversal in the stress field results in the [111] void face producing left handed coils and the [ $[\overline{1} \overline{1}]$ void face producing right handed coils as indicated by the arrows shown at $\tilde{t}=3100$. The opposite coiling directions allow the helical PDLs to displace material in both directions. We note that one full coil displaces the same amount of material as a single PDL.

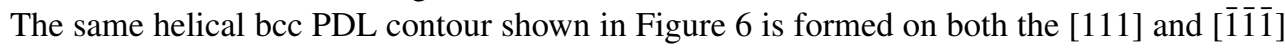
faces of the void over the range of pressures $p=1.0-4.5 \mathrm{GPa}$. The minimum pressure needed to form a helical bcc PDL is 0.5 GPa lower than that needed to form a bcc PDL from an incipient dislocation presented in the previous section. Helical bcc PDLs stop forming below $p<1.0 \mathrm{GPa}$, where image stresses pull the dislocation in toward the center of the void's surface marked by the black " $\times$ ", cf. Figure S6 in the supplementary data for details. The image stress leads 410 to both premature cross-slip and cross-slip onto unexpected secondary slip planes allowing the dislocation to move toward the center of the void's surface. The black " $x$ " is in a region where the resolved shear stresses from the applied load become negligible and the dislocation ceases to move, as indicated by the green region in Figure 1 b). This low pressure regime, where image stress controls cross-slip during helical bcc PDL formation, was not accessible in the incipient 415 loop simulations because of the large pressure needed to stabilize the loop.

Once again, image stresses are shown to have a strong influence on PDL formation in the fcc lattice, shown for two pressures in Figure 7(a) and (b). Helical fcc PDLs are generated for pressures above $p \geq 1.5 \mathrm{GPa}, 1 \mathrm{GPa}$ lower than the pressure needed for an incipient dislocation loop to form an fcc PDL from a void. As previously mentioned, the image stress produces a

420 force directed toward the center of the void's surface as indicated by the orange and cyan arrows labeled $f_{\theta 1,2}$ in Figure 7 (a). In the fcc lattice, the component of the image force projected onto the secondary glide plane depends on the relative angle between the secondary and primary glide planes. The image force at the acute angled corner of the PDL shown by the cyan arrow labeled $f_{\theta 1}$ is closely aligned with the secondary slip plane leading to premature cross-slip. The image

${ }_{425}$ force at the obtuse corner shown by the orange arrow labled $f_{\theta 2}$ is nearly perpendicular to the secondary glide plane and has almost no affect on cross-slip. Premature cross-slip at the corner with the acute angle causes the PDL contour to become compressed along one of its axes as shown in Figure 77a). As the pressure is increased, the effect of image stress on premature cross- 
slip is reduced and the helical fcc PDL contour becomes larger as shown for $p=3.5 \mathrm{GPa}$ in ${ }_{430}$ Figure 7 (b). These image effects are in agreement with those observed in the formation of fcc PDLs from an incipient dislocation loop presented in the previous section [7].

The results for helical PDL generation in the hcp lattice is shown in Figure $7(\mathrm{c})$ and (d). Helical hcp PDLs are generated for pressures at or above $p=1.0 \mathrm{GPa}$. The helical hcp PDL contours show the same pressure dependence and image effects as those presented in the previous 435 section for PDL generation from an incipient loop. For pressures in the range $p=1.5-2.5 \mathrm{GPa}$, helical coiling leads to hexagonal shaped PDL contours and no dislocation cross-slip onto the prismatic plane is observed as illustrated in Figure 7(c). At higher pressures, $p=3.5-4.5 \mathrm{GPa}$, the resolved shear stress becomes large enough to trigger cross-slip from the pyramidal to prismatic plane, producing the eight sided PDL contour shown in Figure 77d). Additional helical 440 PDL contours generated from a void over a range of pressures are shown in Figure S6 of the supplementary data.

PDL generation through helical coiling around a misfit particle is presented in Figure 8 for the bcc lattice. A helical bcc PDL forms through a series of cross-slip events in much the same way as it was formed around the void. Again, the main differences between the void and misfit 445 particle simulations is the absense of a free surface and the presence of a dislocation inside the misfit particle. In order to initiate coiling around the misfit particle, the resolved shear stress near the interface must be strong enough to cause the dislocation to overcome its local line-tension force and bow out. In our simulations, this interaction inhibits the misfit particle from forming full helical PDLs for $p<1.5 \mathrm{GPa}$. Once coiling commences, an internal and external coil will

450 form as shown in Figure 8(a). Consequently, the production of one full helical PDL from each face produces two full helical PDLs inside the misfit particle. The elastic stress field created by the misfit particle forces the interior coils to remain within the region occupied by the misfit particle. The confinement of the interior coils produces strong interactions with one another and with those dislocations forming helical PDLs on the misfit particle's exterior.

455 The helical bcc PDL shown in Figure 8 6 b) is formed at a misfit strain equivalent to $p=$ 1.5 GPa. This is the lowest misfit strain that we found helical PDLs to form in any lattice for this size of misfit particle, $R_{v}=100|\mathbf{b}|$. The helical bcc PDL shows the back and forth cross-slip common to the misfit particle simulations. As the equivalent pressure is increased, the amount of back and forth cross-slipping is reduced. Helical coiling around the misfit particle in the bcc,

$460 \mathrm{fcc}$ and hcp lattices produces nearly ideal PDL contours that do not show a pressure dependence. They are similar to the misfit particle simulations produced from an incipient dislocation loop presented in the previous section.

In several of the helical coiling simulations, the radius of the helical coil increases as the formation progresses. This behavior is observed for all lattices, but is most prominent in the 465 hcp lattice. There are two probable causes for it: (1) the helical coil forming inside the misfit particle pushes the externally forming coils out and (2) the availability of a screw dislocation at the moment cross-slip is favored by the resolved shear stress. If the dislocation contains an edge component, cross-slip becomes delayed until the dislocation reaches a screw orientation. This delay in cross-slip causes the dislocation to move further out on its current glide plane, resulting in the observed outward spiraling of the helical PDL. There is no competing mechanism in the misfit particle simulations to attract the dislocation back toward the center of the misfit's surface. The void simulations do not show this effect as no internal helical coil forms inside the void and the image stresses produced by the void induce premature cross-slip causing the screw dislocation to spiral in towards the center as the helical PDL is formed. Further details on helical coiling around a misfit particle for all pressures and lattice types are presented in Figure S6 of 
the supplementary data.

\subsection{PDL formation time}

In the previous two sections, we described the factors that affect PDL formation over a range of pressures by examining the PDL's final contour. In this section, we examine the effect of 480 those factors (lattice type, defect type, initial dislocation configuration and pressure) on the PDL formation time, $t_{f}$.

The nondimensionalized formation time, $\tilde{t}_{f}$, as a function of pressure for the PDL contours shown in Figures 38 are plotted on a log-log axis in Figure 9, where $\tilde{t}_{f}=t_{f} \mu / B_{\text {screw }}$ is the nondimensionalized time used in other PDL generation studies [9]. Tabulated values for $\tilde{t}_{f}$ and

${ }_{485} t_{f}$ can be found in Tables S3 and S4 of the supplementary data. The PDL formation times as a function of pressure are fit to a power law curve according to $\tilde{t}_{f}=c p^{k}$, where $k$ is the scaling exponent. The value of $k$ is labeled in the figure. If $\tilde{t}_{f}$ were only dependent on pressure, a linear mobility function would predict an inverse relationship between PDL formation time and pressure $(k=-1)$, indicated by the black triangle in Figure 9(a). In contrast, the actual 490 nondimensionalized PDL formation time as a function of pressure decreases faster than $k=-1$ due to the elastic and line-tension interactions between dislocations. The dislocation-dislocation interactions show a stronger pressure dependence than the image stresses as evidenced by the larger decrease in misfit particle PDL formation times with increased pressure when compared to voids.

The actual PDL formation time, $t_{f}$, is related to the nondimensionalized formation time, $\tilde{t}_{f}$, through a mobility function determined from atomistic simulations of dislocations in aluminum [26]. Section S4 of the supplementary data provides details on converting $\tilde{t}_{f}$ to $t_{f}$ using a linear mobility function derived from the high shear stress dislocation mobility data. Actual PDL formation times using the conversion described in section S4 of the supplementary data are presented in Table 1. All PDL formation times are less than $100 \mathrm{ps}$, even at the lowest helical PDL formation pressure of $1.0 \mathrm{GPa}$. To accurately model the PDL formation process in a DD simulation, one would require timesteps of $\approx 1$ ps which are prohibitively small for realistic simulations of strain hardening. However, the DD simulations of PDL formation in this work do provide a timescale that can be used as a rule for introducing PDLs into a DD simulation without explicitly modeling their formation.

The void's growth rate can now be estimated by means of the PDL formation times. Each PDL removes a platelet of material whose thickness is a single Burgers vector, $|\mathbf{b}|$, with a contour approximated by a circle of radius $R_{P D L}=R_{v} / \sqrt{2}$, where $R_{v}$ is the void's radius. Therefore, the volume contained in each platelet is given by $V_{P D L}=\pi R_{P D L}^{2}|\mathbf{b}|$ and each PDL increases the void's volume by $\approx 4 \%$. The growth rate is then given by $\dot{V}=n V_{P D L} / t_{f}$, where $n$ is the number of PDLs being generated at a time and is dependent on the crystal lattice and mode of PDL generation.

The two modes of PDL generation described in sections 3.1 and 3.2 lead to two separate types of void growth. In the dislocation nucleation case, the stress level is sufficient to nucleate multiple dislocations around the void's surface, leading to isotropic void growth in the fcc and bcc lattice. For isotropic void growth in the hcp lattice, other families of Burgers vectors need to be considered. For dislocation nucleation, $n$ is equal to $2 \mathrm{x}$ the number of Burgers vectors in the crystal lattice which would be 12, 8, and 6 for fcc, bcc, and hcp lattices, respectively. The factor of 2x is employed as PDLs are formed in the positive and negative direction of each Burgers vector. Using these values for $n$ neglects the formation of dislocation junctions and locks [32, 33], which could render some sites for PDL generation inoperable. On the other hand, only a single source for PDL generation is available through helical coiling of a screw dislocation, resulting in 
cylindrical growth of the void in the directions of the dislocation's Burgers vector where $n=2$. The PDL formation times given in Table 1 are then used with these values for $n$ to predict the void growth rates given in Table 2 for a void with $R_{v}=100|\mathbf{b}|$.

Three trends are apparent in Figure 6 and the data in Tables 1 and 2 . The first trend is that PDLs form faster in bcc and hcp lattices than those in an fcc lattice. As described in sections 3.1 3.2 and S2 of the supplementary data, the dislocation forming the PDL experiences larger resolved shear stress in the bcc and hcp lattice due to an increased number of slip-systems available for cross-slip when compared to the fcc lattice. However, for the case of dislocation nucleation from a void, the void's growth rate reflected by the data in Table 2 is actually slowest for the hcp lattice due to the limited number of $\langle a\rangle$-type Burgers vectors available for PDL generation. The second trend is that PDLs form faster around voids than misfit particles even though the resolved shear stress created by the applied load is identical for both defect types. This difference is most likely due to the PDLs being created inside the misfit particle. The internal PDLs increase the dislocation-dislocation interactions with the PDLs forming on the particle's exterior. The third trend is that incipient dislocation loops form PDLs faster than infinite screw dislocations. This is due to incipient loops having two surface piercing dislocations traveling around the PDL contour. Therefore each arm of the loop only has to traverse half the PDL contour whereas the single arm of the infinite screw dislocation must traverse the entire loop. This leads to incipient loops forming PDLs twice as fast as infinite screw dislocations for bcc and hcp lattices in Table 1 . This difference is increased to a factor of 5 to $10 \mathrm{x}$ when the void growth rate given in Table 2 is considered.

\section{Conclusion}

We have carried out a systematic study of PDL formation around spherical defects as a function of pressure. We have considered face-centered cubic (fcc), body-centered cubic (bcc) and hexagonal close-packed (hcp) lattices, void and misfit particle spherical defects, and two initial dislocation configurations. The first initial dislocation configuration simulated is an incipient dislocation loop corresponding to a dislocation nucleated from the defect surface. This is the configuration considered in the classical model of PDL formation by Ashby and Johnson [2].

550 The second initial dislocation configuration consists of an infinite screw dislocation intersecting the void. This configuration corresponds to a lattice with an existing dislocation content. The infinite screw dislocation produces a PDL in the form of a helical dislocation coil, where each coil removes the same amount of material as a single PDL. The infinite screw dislocation is able to form PDLs continuously at lower pressures without the need for dislocation nucleation.

The highest rates of PDL generation are for a dislocation nucleated from a void in a bcc or hcp lattice. The increased PDL formation rate in bcc and hcp, as opposed to fcc, is primarily due to their extra glide planes available for cross-slip. However, the void growth rate for nucleated dislocations was lowest in the hcp lattice due to the limited number of $\langle a\rangle$-type Burgers vectors available for PDL generation. Voids increase the PDL formation rate when compared to misfit particles by cutting the dislocation at the surface. PDLs form around voids without having to compete with PDL formation inside the misfit particle. The incipient dislocation loop decreases PDL formation time over the infinite screw initial configuration by raising the number of surface piercing dislocations encircling the void. This allows for the creation of a full PDL after each surface piercing dislocation circles half the PDL contour, thus cutting PDL formation time in half. The formation time for a full PDL is found to be $15-85$ ps. 
As shown in our previous work [7], the fcc lattice with a void is susceptible to image induced cross-slip. Both the incipient loop and infinite screw dislocation in the fcc lattice display this behavior. As the pressure is increased, the effect of image stress on cross-slip is diminished. This yields two distinct pressure regimes for the incipient dislocation loop in the fcc lattice. For the infinite screw dislocation this transition from image- to pressure-dominated cross-slip occurs gradually with increasing pressure. Conversely, image effects play a minor role in the formation of full PDLs in the bcc and hep lattices for both initial dilsocation configurations. At low pressures, the image effects cause PDL formation to cease in the bcc and hcp lattices for the infinite screw dislocation. If it were not for image stresses, the infinite screw dislocation would continuously produce helical PDLs from a void at any pressure.

\section{Acknowledgments}

Support of the U.S. Army Research Laboratory's Enterprise for Multiscale Research of Materials is gratefully acknowledged. Computing support was provided by the DoD Supercomputing Resource Center located at the U.S. Army Research Laboratory.

[1] M. Ashby, The deformation of plastically non-homogeneous materials, Philosophical Magazine 21 (170) (1970) 399-424.

[2] M. Ashby, L. Johnson, On the generation of dislocations at misfitting particles in a ductile matrix, Philosophical Magazine 20 (167) (1969) 1009-1022.

[3] T. Tsuru, Y. Shibutani, Initial yield process around a spherical inclusion in single-crystalline aluminium, Journal of Physics D: Applied Physics 40 (7) (2007) 2183.

[4] Y. Tang, E. Bringa, B. Remington, M. Meyers, Growth and collapse of nanovoids in tantalum monocrystals, Acta Materialia 59 (4) (2011) 1354-1372.

[5] S. Rao, D. Dimiduk, T. Parthasarathy, M. Uchic, C. Woodward, Atomistic simulations of surface cross-slip nucleation in face-centered cubic nickel and copper, Acta Materialia 6 (7) (2013) 2500-2508.

[6] A. Hussein, S. Rao, M. Uchic, D. Dimiduk, J. El-Awady, Microstructurally based cross-slip mechanisms and their effects on dislocation microstructure evolution in fcc crystals, Acta Materialia 85 (2015) 180-190.

[7] L. B. Munday, J. C. Crone, J. Knap, The role of free surfaces on the formation of prismatic dislocation loops, Scripta Materialia 103 (2015) 65-68.

[8] B. Makenas, H. Birnbaum, Phase changes in the niobium-hydrogen system I: Accommodation effects during hydride precipitation, Acta Metallurgica 28 (7) (1980) 979-988.

[9] P. Geslin, B. Appolaire, A. Finel, Investigation of coherency loss by prismatic punching with a nonlinear elastic model, Acta Materialia 71 (2014) 80-88.

[10] S. Amelinckx, W. Bontinck, W. Dekeyser, F. Seitz, On the formation and properties of helical dislocations, Philosophical Magazine 2 (15) (1957) 355-378.

600 [11] D. Rodney, Molecular dynamics simulation of screw dislocations interacting with interstitial frank loops in a model FCC crystal, Acta materialia 52 (3) (2004) 607-614

[12] T. Nogaret, D. Rodney, M. Fivel, C. Robertson, Clear band formation simulated by dislocation dynamics: Role of helical turns and pile-ups, Journal of Nuclear Materials 380 (1) (2008) 22-29.

[13] E. Van der Giessen, A. Needleman, Discrete dislocation plasticity: a simple planar model, Modelling and Simulation in Materials Science and Engineering 3 (5) (1995) 689.

[14] J. Segurado, J. Llorca, An analysis of the size effect on void growth in single crystals using discrete dislocation dynamics, Acta Materialia 57 (5) (2009) 1427-1436.

[15] H.-J. Chang, J. Segurado, J. Llorca, Three-dimensional dislocation dynamics analysis of size effects on void growth, Scripta Materialia 95 (2015) 11-14.

[16] C. Weinberger, W. Cai, Surface-controlled dislocation multiplication in metal micropillars, Proceedings of the National Academy of Sciences 105 (38) (2008) 14304-14307.

[17] J. Greer, C. Weinberger, W. Cai, Comparing the strength of fcc and bcc sub-micrometer pillars: Compression experiments and dislocation dynamics simulations, Materials Science and Engineering: A 493 (1) (2008) 21-25.

[18] R. Rudd, Void growth in bcc metals simulated with molecular dynamics using the Finnis-Sinclair potential, Philosophical Magazine 89 (34-36) (2009) 3133-3161.

[19] N. Chaari, E. Clouet, D. Rodney, First-Principles Study of Secondary Slip in Zirconium, Physical review letters 112 (7) (2014) 075504 
[20] A. Arsenlis, W. Cai, M. Tang, M. Rhee, T. Oppelstrup, G. Hommes, T. Pierce, V. Bulatov, Enabling strain hardening simulations with dislocation dynamics, Modelling and Simulation in Materials Science and Engineering 15 (6) (2007) 553.

[21] K. Leiter, J. Crone, J. Knap, An algorithm for massively parallel dislocation dynamics simulations of small scale plasticity, Journal of Computational Science 4 (5) (2013) 401-411.

[22] J. Crone, P. Chung, K. Leiter, J. Knap, S. Aubry, G. Hommes, A. Arsenlis, A multiply parallel implementation of finite element-based discrete dislocation dynamics for arbitrary geometries, Modelling and Simulation in Materials Science and Engineering 22 (3) (2014) 035014

[23] P. Amestoy, I. Duff, J.-Y. L'Excellent, Multifrontal parallel distributed symmetric and unsymmetric solvers, Computer methods in applied mechanics and engineering 184 (2) (2000) 501-520.

[24] J. Crone, L. Munday, Parallel Performance of Linear Solvers and Preconditioners, Tech. Rep., Army Research Laboratory, 2014.

25] W. Cai, A. Arsenlis, C. Weinberger, V. Bulatov, A non-singular continuum theory of dislocations, Journal of the Mechanics and Physics of Solids 54 (3) (2006) 561-587.

[26] D. Olmsted, L. Hector Jr, W. Curtin, R. Clifton, Atomistic simulations of dislocation mobility in $\mathrm{Al}, \mathrm{Ni}$ and $\mathrm{Al} / \mathrm{Mg}$ alloys, Modelling and Simulation in Materials Science and Engineering 13 (3) (2005) 371

[27] S. Aubry, M. Rhee, V. Bulatov, G. Hommes, A. Arsenlis, Dislocation Dynamics in hexagonal close-packed crystals, Journal of the Mechanics and Physics of Solids Xxx (xxx) (submitted for publication (2015)) Xxx-Xxx.

[28] H. Fan, S. Aubry, A. Arsenlis, J. A. El-Awady, The role of twinning deformation on the hardening response of polycrystalline magnesium from discrete dislocation dynamics simulations, Acta Materialia 92 (2015) $126-139$.

[29] D. Weygand, L. Friedman, E. Van der Giessen, A. Needleman, Aspects of boundary-value problem solutions with three-dimensional dislocation dynamics, Modelling and Simulation in Materials Science and Engineering 10 (4) (2002) 437.

[30] J. Eshelby, The determination of the elastic field of an ellipsoidal inclusion, and related problems, Proceedings of the Royal Society of London. Series A. Mathematical and Physical Sciences 241 (1226) (1957) 376-396.

[31] S. Groh, E. Marin, M. Horstemeyer, D. Bammann, Dislocation motion in magnesium: a study by molecular statics and molecular dynamics, Modelling and Simulation in Materials Science and Engineering 17 (7) (2009) 075009.

645 [32] J. Marian, J. Knap, M. Ortiz, Nanovoid cavitation by dislocation emission in aluminum, Physical review letters 93 (16) (2004) 165503.

[33] M. Ponga, M. Ortiz, M. Ariza, Finite-temperature non-equilibrium quasi-continuum analysis of nanovoid growth in copper at low and high strain rates, Mechanics of Materials xxx (xxx) (in press 2015) xxx-xxx, doi 10.1016/j. mechmat.2015.02.007 


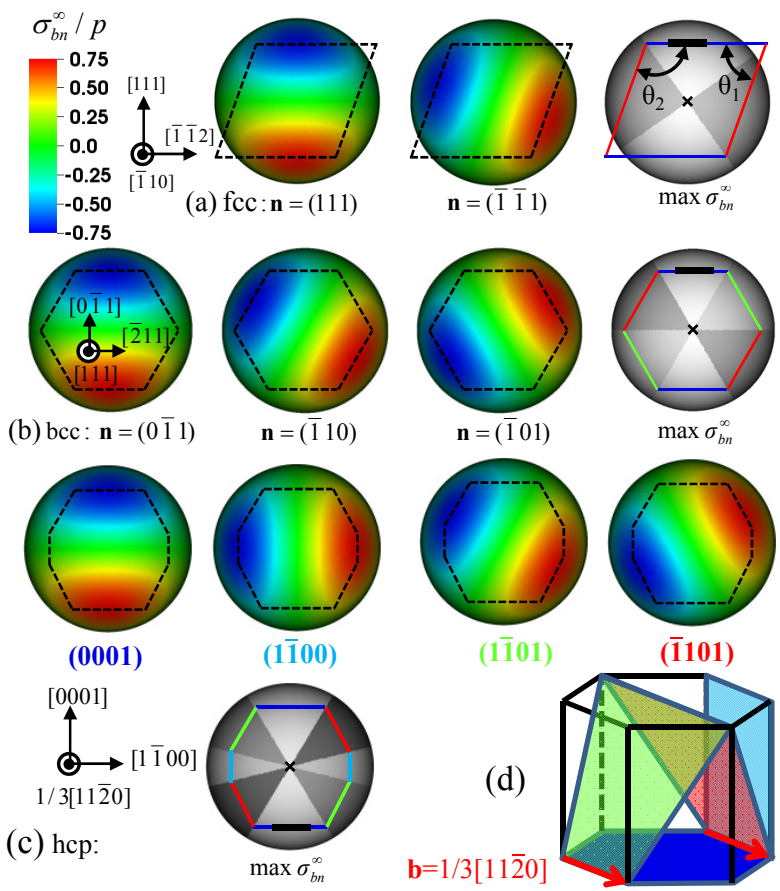

Figure 1: Resolved shear stress, $\sigma_{b n}^{\infty}$, near the void surface due to the far-field hydrostatic load for (a) fcc glide planes sharing $\mathbf{b}=$ [110], (b) bcc glide planes sharing $\mathbf{b}=$ [111], and (c) hcp glide planes sharing the $\langle a\rangle$-type dislocation with $\mathbf{b}=\frac{1}{3}[11 \overline{2} 0]$. (d) An illustration of the hcp lattice with PDL glide planes colored to match the glide plane labels in (c) with basal in blue, prismatic in cyan, and the two type-I pyramidal planes in green and red. The color bar shows the normalized stress, $\sigma_{b n}^{\infty} / p$. The dashed black lines indicate the ideal PDL contour that maximizes $\sigma_{b n}^{\infty}$ on each plane. The final gray scale figure shows the void surface colored by the glide plane, $\mathbf{n}$, that maximizes $\left|\sigma_{b n}^{\infty}\right|$. The line colors of the ideal PDL contour indicate the dislocation's different glide planes. The solid black line indicates the location and size of the incipient dislocation loop. 


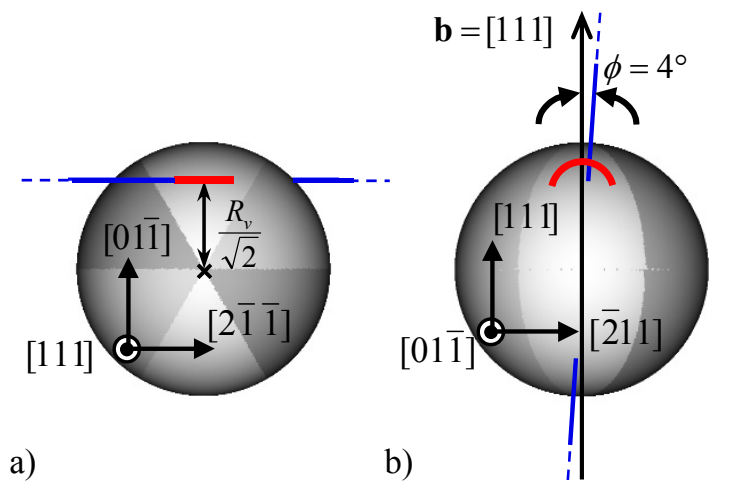

Figure 2: Initial dislocation configurations for the incipient dislocation loop and infinite screw dislocation in a bcc crystal. The screw dislocation is shown by the blue lines. The solid blue line indicates the portion of the dislocation being modeled in ParaDiS. The dashed blue lines are to indicate the dislocation being extended to infinity. The incipient dislocation loop is shown by the red line and is most easily observed by the half loop in (b). Gray scale coloring of void surface indicates the glide plane that maximizes the resolved shear stress as described in Figure 1p. 


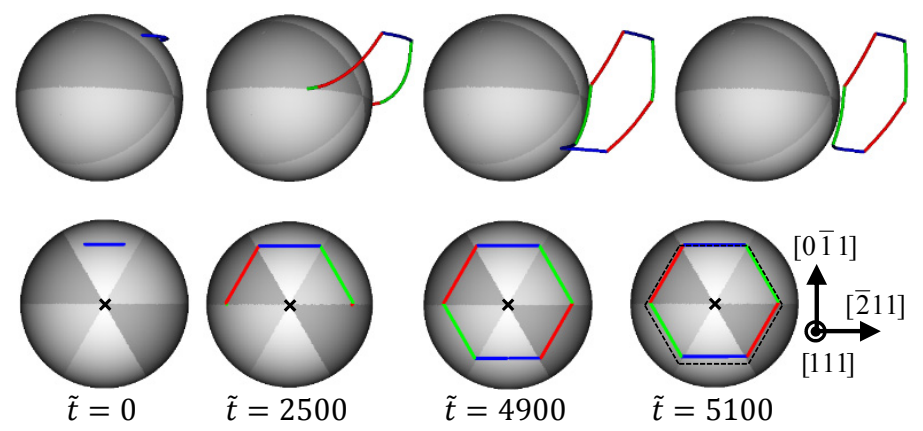

Figure 3: Generation of a prismatic dislocation loop (PDL) in a bcc lattice for $p=2.5 \mathrm{GPa}$. The dislocation is colored according to its glide plane where blue, red, and green correspond to $\mathbf{n}=(0 \overline{1} 1),(\overline{1} 10)$ and $(\overline{1} 01)$, respectively. The black dashed hexagon for $\tilde{t}=7.0$ indicates the ideal PDL contour for a bcc lattice. The gray scale coloring of the void surface indicates the glide plane that maximizes $\left|\sigma_{b n}^{\infty}\right|$ as described in Figure 1 . 


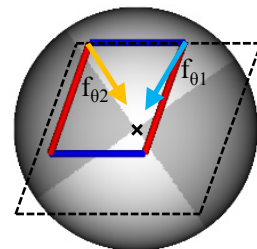

a) fcc Void $p=2.5 \mathrm{GPa}$ $\tilde{t}=45900$

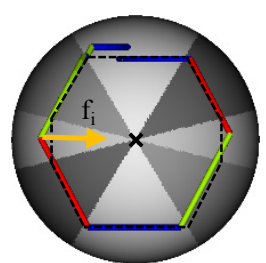

d) hep Void

$p=2.5 \mathrm{GPa}$

$\tilde{t}=6700$

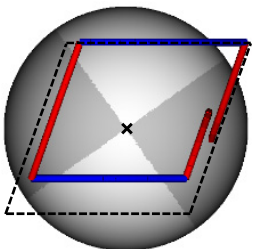

b) fcc Void

$$
p=4.5 \mathrm{GPa}
$$$$
\tilde{t}=5000
$$

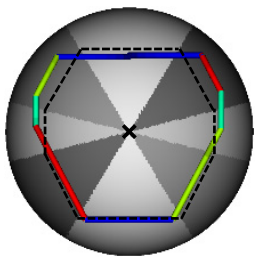

e) hep Void
$p=4.0 \mathrm{GPa}$
$\tilde{t}=3400$

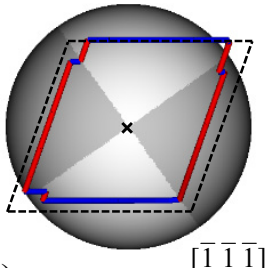

c) fcc Misfit $p=2.5 \mathrm{GPa}$ $\tilde{t}=50500$

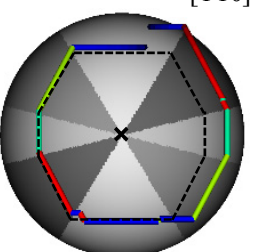

f) hcp Misfit

[0001]

$p=2.5 \mathrm{GPa}$

$\tilde{t}=24700$

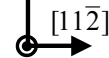

[110]

Figure 4: Final PDL contours created by an incipient dislocation loop for (a)-(c) an fcc lattice and (d)-(f) an hcp lattice. The ideal PDL contour is shown by the black dashed line. The dislocation of the actual PDL contour is colored according to its glide plane. For the fcc lattice, blue and red correspond to $\mathbf{n}=(111)$, and (1111), respectively. For the hcp lattice, blue and cyan correspond to basal and prismatic slip, respectively, while red and green correspond to slip on the two type-I pyramidal planes. The orange and cyan colored arrows in (a) and (d) represent the direction of the image force at the location of cross-slip. The gray scale coloring of the void surface indicates the glide plane that maximizes $\left|\sigma_{b n}^{\infty}\right|$ as described in Figure 1 

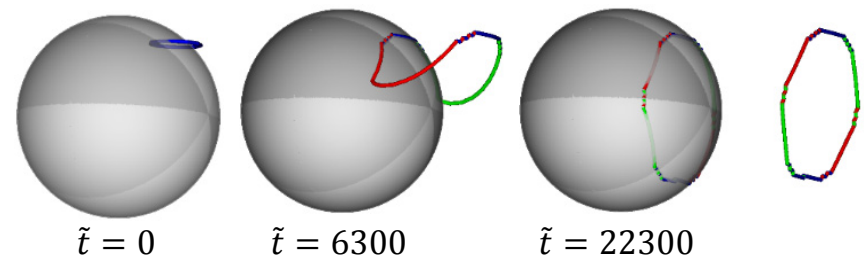

a)

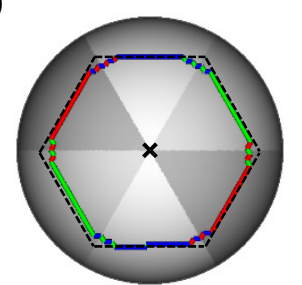

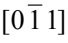

b) $\tilde{t}=15000$

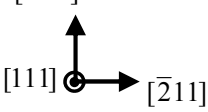

Figure 5: a) Generation of a PDL from a misfit particle in the bcc lattice for a dilational misfit strain equivalent to $p=2.5 \mathrm{GPa}$. In these simulations the dislocation is not cut at the misfit particle interface but extends inside it, as indicated by the slight gray coloring of the dislocation line. b) The final bcc misfit particle PDL contour formed at $\tilde{t}=15000$ shows rounded corners due to multiple back and forth cross-slip events. The dislocation and misfit particle coloring are described in Figures 1 and 3 


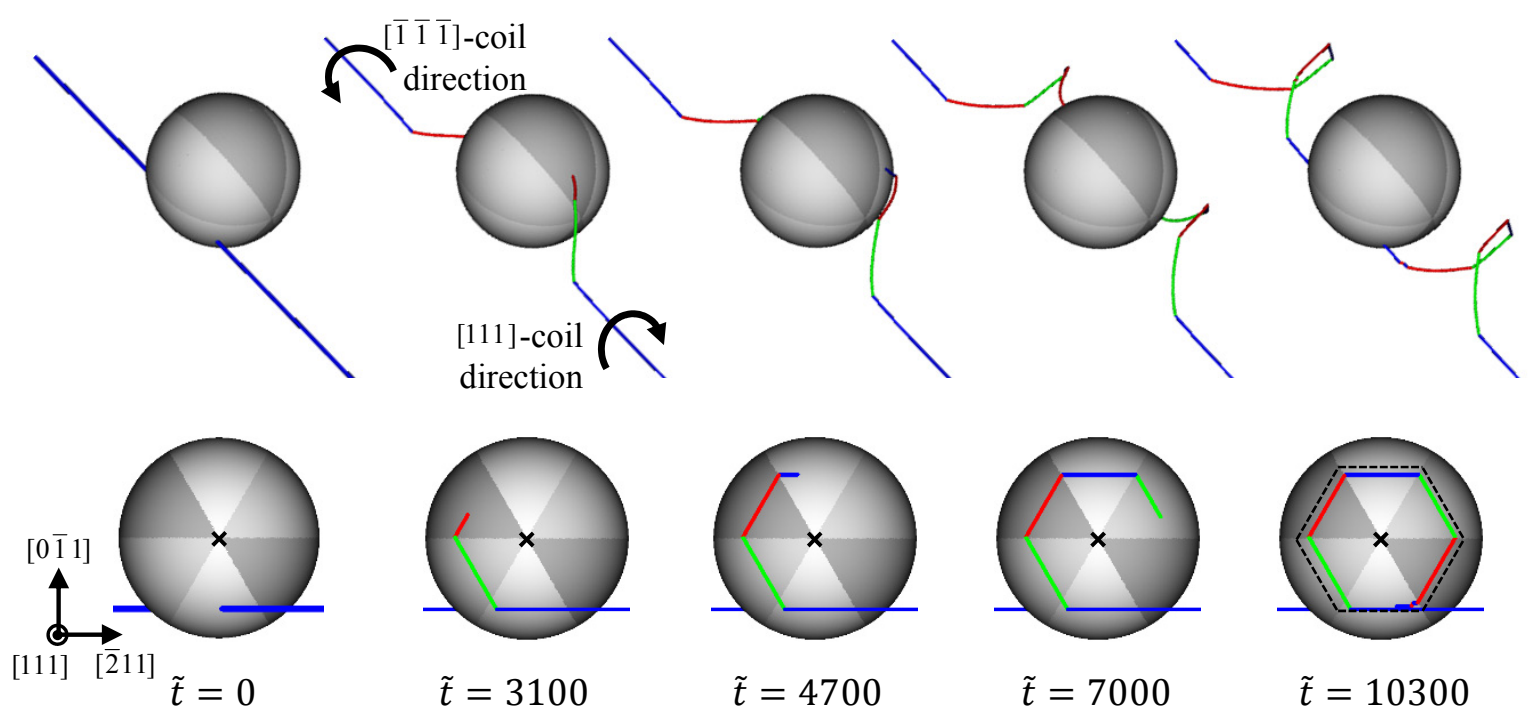

Figure 6: Helical PDL formation through coiling around a void in a bcc lattice at $p=2.5 \mathrm{GPa}$. The dislocation is colored according to its glide plane where blue, red, and green correspond to $\mathbf{n}=(0 \overline{1} 1),(\overline{1} 10)$ and $(\overline{1} 01)$, respectively. The black dashed hexagon for $\tilde{t}=10300$ indicates the ideal PDL contour for a bcc lattice. The gray scale coloring of the void surface indicates the glide plane that maximizes $\left|\sigma_{b n}^{\infty}\right|$ as described in Figure 1 p. 


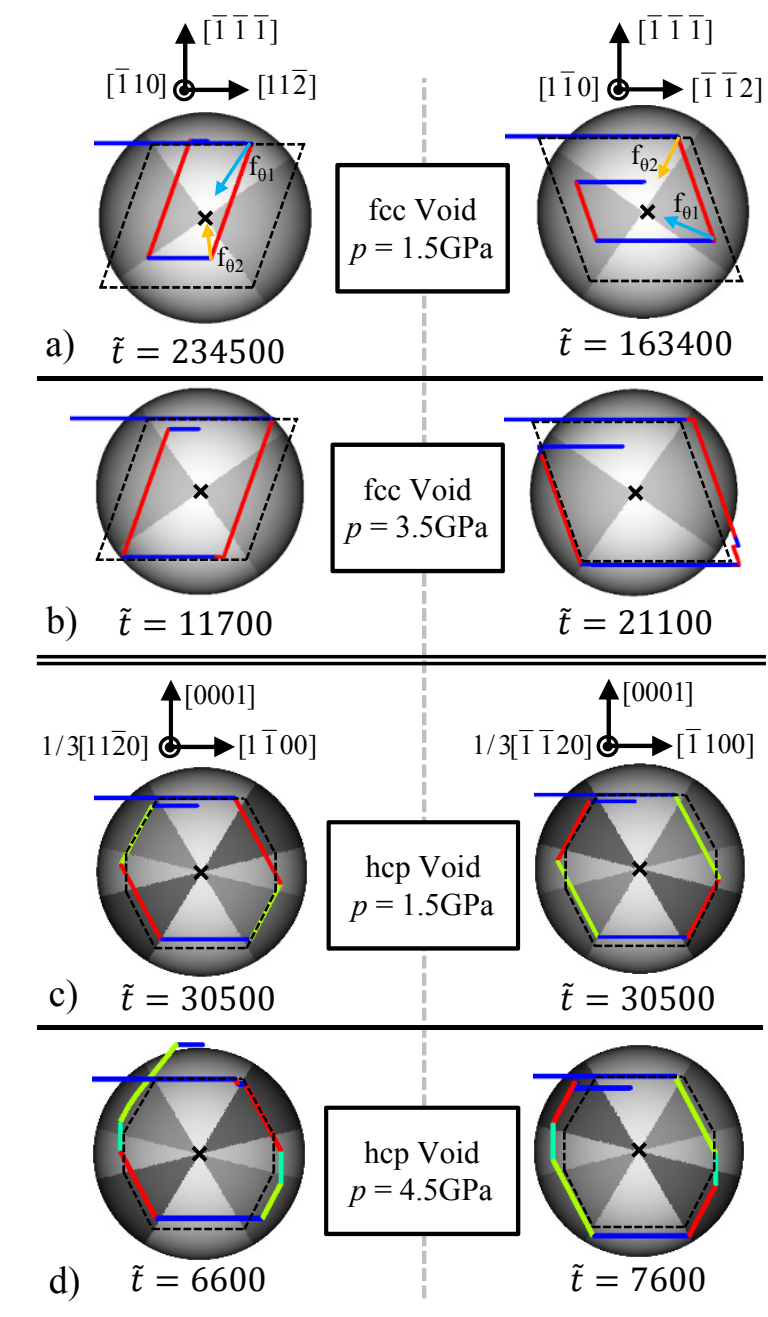

Figure 7: Helical PDL contours for void simulations in (a),(b) an fcc lattice and (c),(d) an hcp lattice. Two helical PDLs are shown for each pressure for the PDL formed on the front and back face of the void as indicated by the axes. The orange and cyan colored arrows in (a) represent the direction of the image force at the location of cross-slip. Dislocation and void coloring are described in Figure 1 


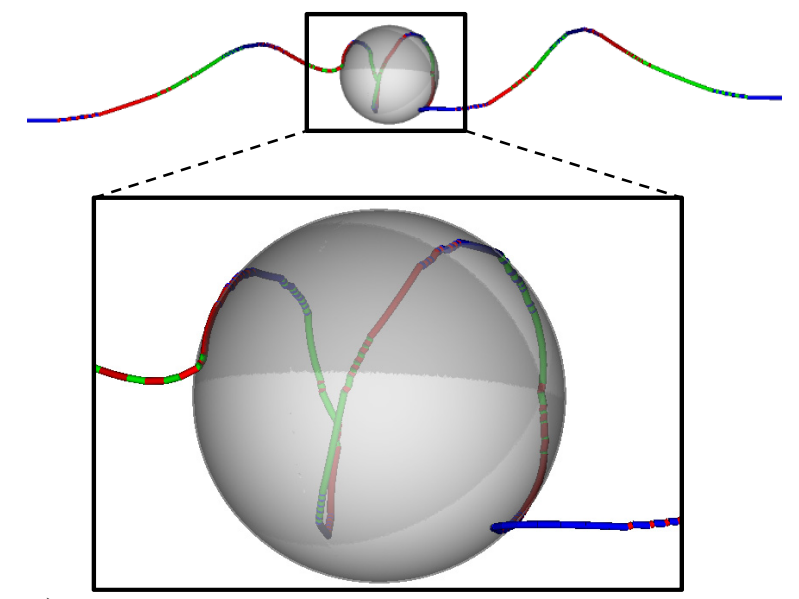

a)

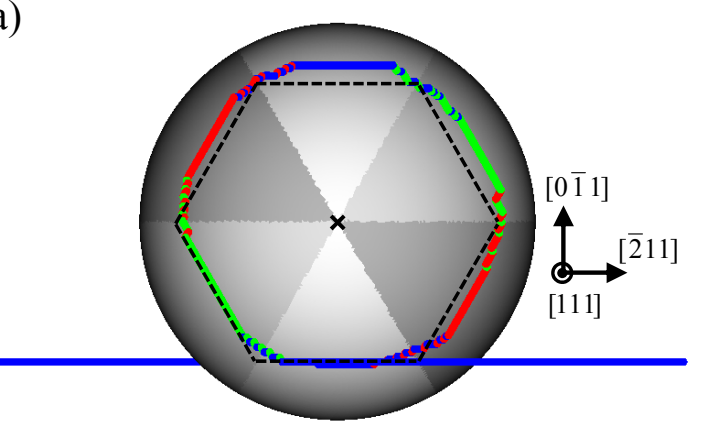

b)

$$
\tilde{t}=194400
$$

Figure 8: Helical bcc PDL formed around a misfit particle that produces an equivalent pressure of $p=1.5 \mathrm{GPa}$. (a) Profile of the helical coils that form exterior and interior to the particle. One helical coil forms from each face of the misfit, resulting in two full helical coils formed interior to the misfit particle. (b) Final helical bcc PDL contour. 


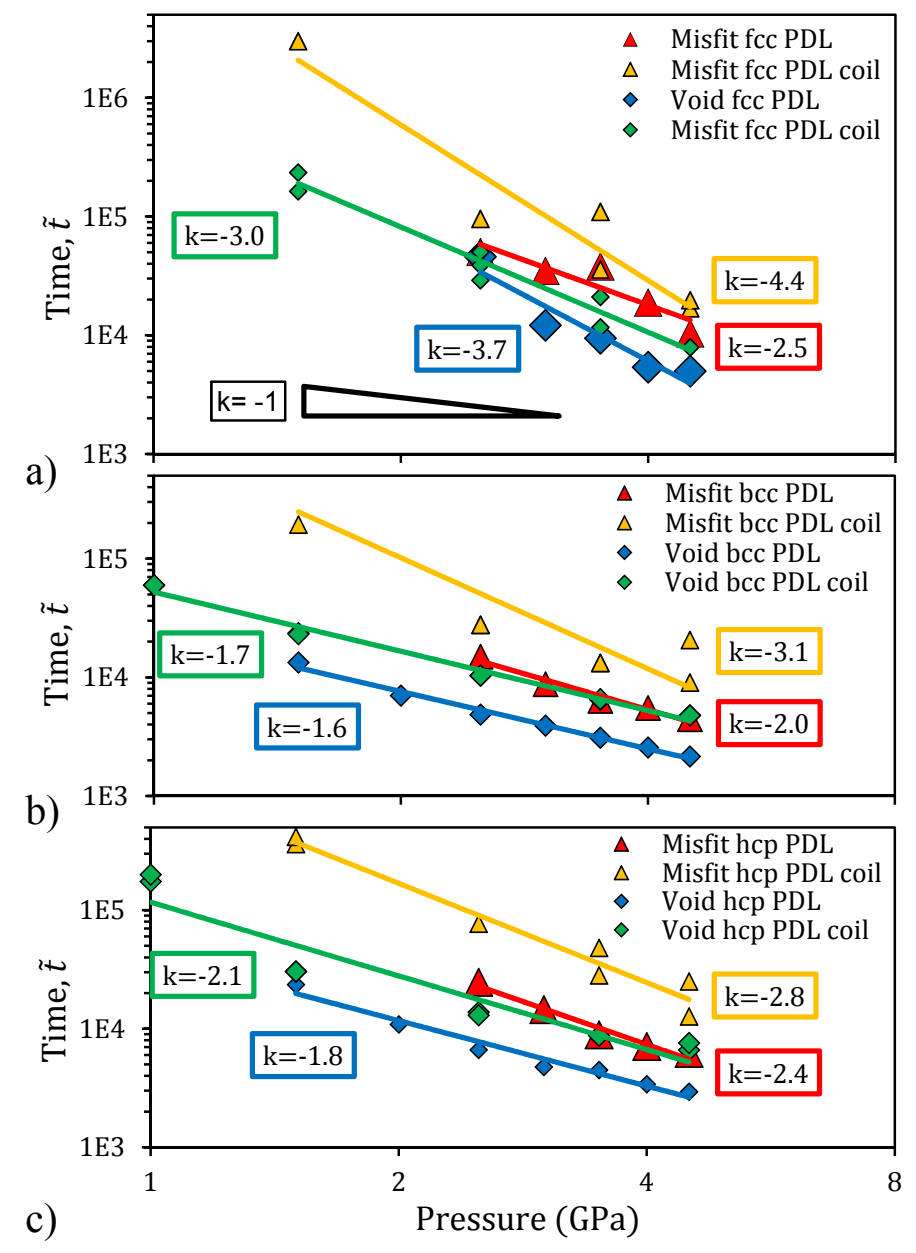

Figure 9: Formation time of first prismatic loop as a function of pressure for (a) fcc, (b) bcc, and (b) hcp slip systems. Lines are power law curve fits to the data, $\tilde{t}_{f}=c p^{k}$, where the fitting exponent $k$ is labeled on the plots with box color matching the color of the data points. Numerical values for the data points are tabulated in Table S3 of the supplementary data. 
Table 1: PDL formation time, $t_{f}(\mathrm{ps})$, for void defects as a function of hydrostatic tension $p$. Only the first PDL formed from the infinite screw configuration is given.

\begin{tabular}{|c|c|c|c|c|c|c|}
\hline \hline \multirow{2}{*}{$\begin{array}{c}\text { Pressure } \\
(\mathrm{GPa})\end{array}$} & \multicolumn{2}{|c|}{ fcc } & \multicolumn{2}{c|}{ bcc } & \multicolumn{2}{c|}{ hcp } \\
\cline { 2 - 7 } & loop & screw & loop & screw & loop & screw \\
\hline 1.0 & -- & -- & -- & 77.4 & -- & 83.5 \\
1.5 & -- & 83.2 & 34.0 & 65.7 & 37.5 & 69.8 \\
2.5 & 40.2 & 69.2 & 24.5 & 50.4 & 27.8 & 55.2 \\
3.5 & 31.1 & 53.0 & 19.7 & 40.3 & 23.7 & 46.8 \\
4.5 & 24.8 & 44.6 & 15.9 & 33.8 & 19.2 & 40.8 \\
\hline
\end{tabular}


Table 2: Void growth rate $\left(|\mathbf{b}|^{3} / \mathrm{ps}\right)$ of a void with $R_{v}=100|\mathbf{b}|$ as a function of hydrostatic tension $p$. The number of PDLs being simultaneously generated for each lattice type and dislocation source is given by $n$.

\begin{tabular}{|c|c|c|c|c|c|c|}
\hline \hline \multirow{2}{*}{$\begin{array}{c}\text { Pressure } \\
(\mathrm{GPa})\end{array}$} & \multicolumn{2}{|c|}{ fcc } & \multicolumn{2}{c|}{ bcc } & \multicolumn{2}{c|}{ hcp } \\
\cline { 2 - 7 } & loop & screw & loop & screw & loop & screw \\
& $n=2$ & $n=8$ & $n=2$ & $n=6$ & $n=2$ \\
\hline 1.0 & -- & -- & -- & 100 & -- & 90 \\
1.5 & -- & 90 & 930 & 120 & 630 & 110 \\
2.5 & 1170 & 110 & 1280 & 160 & 850 & 140 \\
3.5 & 1510 & 150 & 1600 & 200 & 990 & 170 \\
4.5 & 1900 & 180 & 1980 & 230 & 1230 & 190 \\
\hline
\end{tabular}




\section{Graphical Abstract}

\section{Dislocation mediated void growth through:}

a) Prismatic dislocation loop generation from an incipient dislocation loop
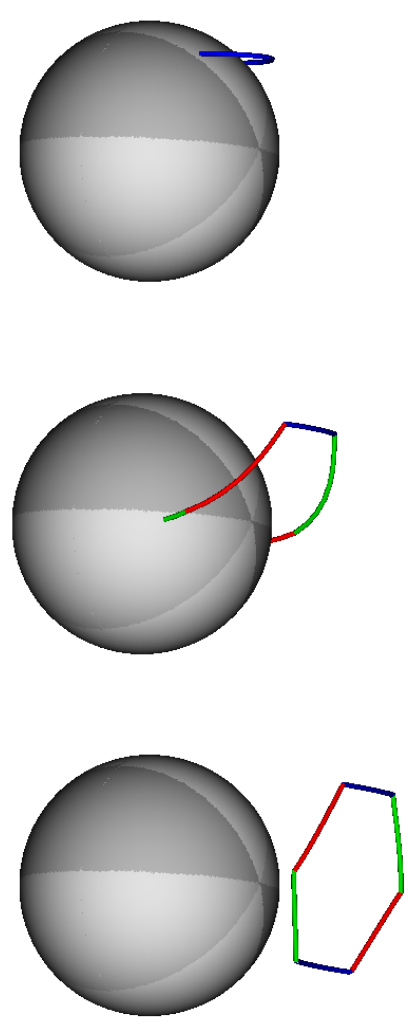

b) Prismatic dislocation loop generation through helical coiling of a screw dislocation.
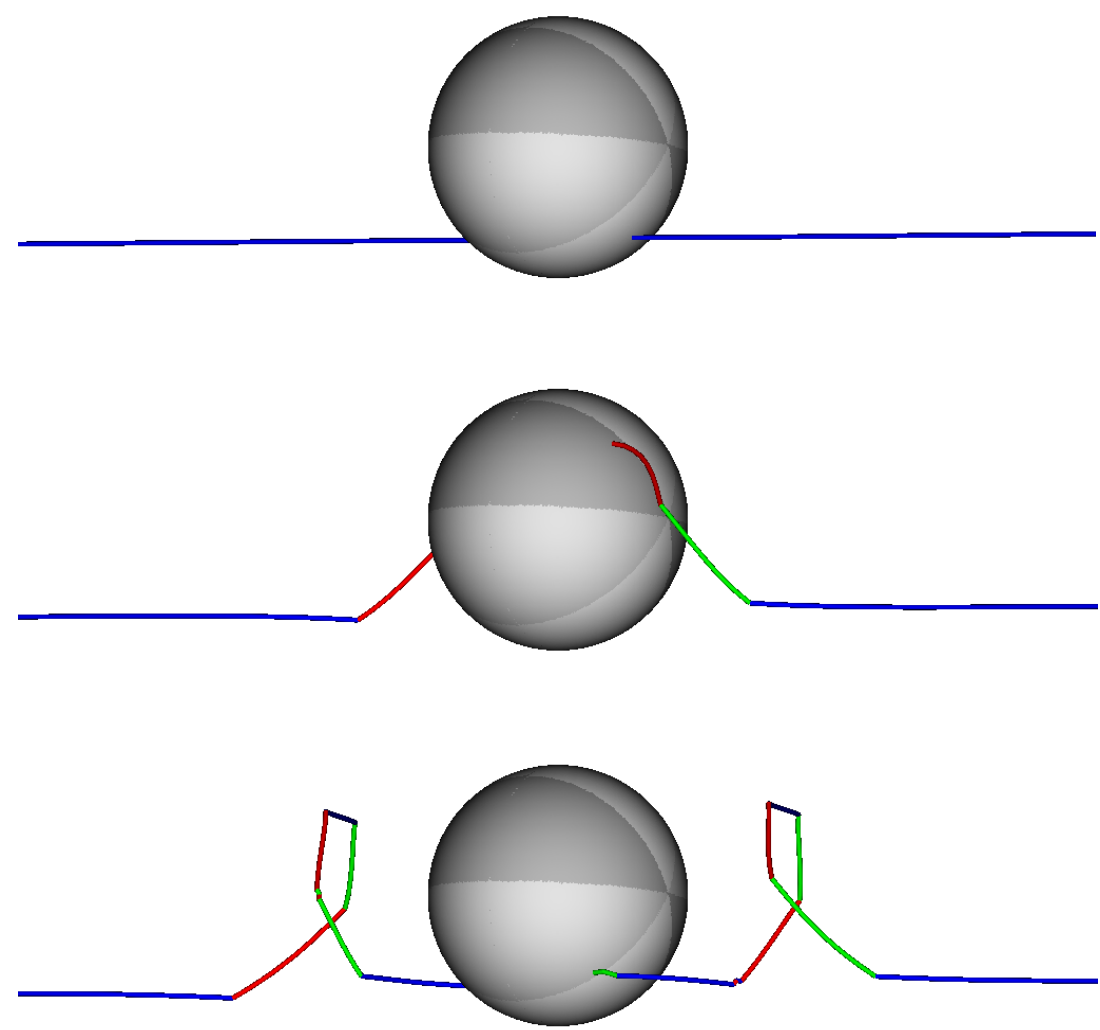